\title{
La terapia a pressione negativa: costi e benefici Negative pressure therapy: costs and benefits
}

\author{
Fiorella Riservati \\ Wound Care Specialist, Distretto Cure Domiciliari, Urbino, Italia
}

\section{RIASSUNTO}

Con questo mio lavoro ho cercato di valutare quali aspetti caratterizzano la terapia a pressione negativa (o negative pressure therapy, NTP) e analizzato la validità di questo presidio mettendolo a confronto con l'utilizzo delle medicazioni avanzate. Oggi, in seguito alla spending review i costi di acquisizione di medicazioni, tubi, contenitori, e il costo del noleggio per uso domiciliare sono più elevati rispetto ad altri tipi di medicazioni, anche se oggi i costi della NTP stanno drasticamente diminuendo.

\section{ABSTRACT}

With this work I tried to evaluate which aspects characterize the negative pressure therapy (NTP) and I analyzed the validity of this technique comparing it with the use of advanced dressings. Today, due to the spending review, the costs of dressings, tubes, containers, and the cost of renting them for home use are higher than other types of dressings, although today the costs of NTP are drastically decreasing.

\section{INTRODUZIONE}

L'avvento della terapia mediante pressione topica negativa ha rivoluzionato la gestione delle lesioni cutanee acute e croniche. Con questo studio, dopo aver consultato vari articoli, vorrei fare capire l'importanza dell'utilizzo della terapia a pressione negativa nel campo del wound care, settore che si sta rinnovando nella gestione delle ferite difficili. Questa mia scelta è maturata durante il mio percorso biennale del master in Wound Care, dove a lezione ho capito l'importanza di questo sistema e il suo uti-

Corrispondenza: Fiorella Riservati, Wound Care Specialist, Distretto Cure Domiciliari, Urbino, Italia.

E-mail: f.riservati@libero.it

Parole chiave: Terapia a pressione negativa; Wound care; Costs; Benefits.

Conflitto d'interesse: l'autore dichiara l'assenza di conflitti d'interesse.

Fondi: nessuno.

Ricevuto per la pubblicazione: 22 May 2018.

Revisione ricevuta: 18 December 2018.

Accettato per la pubblicazione: 18 December 2018 .

This work is licensed under a Creative Commons Attribution NonCommercial 4.0 License (CC BY-NC 4.0).

(C) Copyright F. Riservati, 2018

Licensee PAGEPress, Italy

Italian Journal of Wound Care 2018; 2(3):76-80

doi:10.4081/ijwc.2018.31 lizzo nelle più svariate specialistiche. Il mio elaborato presentato come argomento di tesi si articola in vari capitoli; nell'ultimo capitolo ho cercato di evidenziare le valutazioni economico-sanitarie nell'impiego della negative pressure therapy (NTP) in confronto delle medicazioni avanzate.

La terapia a pressione negativa rappresenta un metodo efficace per la gestione delle ferite e una valida alternativa alle medicazioni tradizionali:

i) Minor frequenza nel cambio della medicazione;

ii) Riduzione dei tempi di guarigione.

Nonostante le nuove tecnologie e medicazioni usate nel trattamento nelle ferite di difficile guarigione siano più costose delle terapie di confronto, il loro uso comunque può essere vantaggioso in termini di costo se porta a una guarigione più veloce ed efficace.

Il costo della gestione delle ferite croniche può dipendere da vari fattori:

i) Frequenza di cambio delle medicazioni e tempo di assistenza necessario;

ii) Percentuali di guarigione;

iii) Impatto sull'ospedalizzazione ed eventi avversi.

Nel sistema a pressione topica negativa il cambio delle medicazioni veniva svolto ogni 4 giorni; mentre con la terapia standard si eseguivano ogni 2 giorni; ciò permette al paziente tranquillità e riduce le sollecitazioni e lo stress sulla ferita.

Controllo dell'essudato: protegge la cute perilesionale dalla macerazione.

Riduzione del rischio di infezione: la sigillatura del sistema ed il minor numero di cambi, riducono la possibilità di contaminazione ed infezione della ferita.

i) Rapida formazione del tessuto di granulazione, epitelizzazione e contrazione della ferita. La NTP stimola 
la formazione di nuovo tessuto. Questo può anche far accrescere la soddisfazione del paziente che può riscontrare risultati positivi in tempi brevi.

ii) Riduzione del dolore nei cambi di medicazione.

iii) Riduzione dell'odore della ferita: una migliore gestione dell'essudato comporta che spesso, nel corso della terapia, l'odore proveniente dalla ferita diminuisca.

iv) Costi di trattamento: una riduzione della frequenza dei cambi di medicazione e una più rapida chiusura della ferita possono favorire la riduzione dei costi generali del trattamento.

\section{ANALISI ECONOMICO-SANITARIA NELL'IMPIEGO DELLA TERAPIA A PRESSIONE TOPICA NEGATIVA}

Oggi circa il $4 \%$ dei costi totali del sistema sanitario è da imputarsi alle ferite come conseguenza dell'innalzamento dell'età media della popolazione ancora in crescita. $^{1}$

In Italia il trend di invecchiamento sta interessando la popolazione italiana e questo comporterà una crescita media dell' $8 \%$ delle ulcere croniche nei prossimi 5 anni.

Le lesioni dell'arto inferiore costituiscono un impegno pari al 56\% delle medicazioni eseguite in ambito domiciliare.

Questi interventi occupano dal 30 al 50\% del tempo totale dell'infermiere domiciliare e il tempo medio per le medicazioni (esclusi gli spostamenti), è di circa 2025 minuti.

Negli ospedali italiani, il $25-50 \%$ dei posti letto è occupato da pazienti con ferite e in ambito domiciliare la gestione delle lesioni cutanee occupa più della metà delle risorse.

E logico che, in tempi di spending review, l'esame delle spese sostenute dallo stato per il funzionamento dei suoi uffici e per la fornitura di servizi ai cittadini, ha lo scopo di ridurre gli sprechi e di apportare miglioramenti al bilancio.

Negli ultimi 10 anni, la ricerca sui costi sanitari correlati al mondo del Wound Care, ha dimostrato che la medicazione acquistata a minor costo è risultata poi la più costosa nel processo di guarigione.

Dal punto di vista economico, una medicazione che rimane in situ più di altre, permette cambi meno frequenti con una riduzione dei costi d'acquisto della medicazione stessa, del tempo infermieristico, del rischio d'infezione della ferita stessa.

Il tempo del personale infermieristico economicamente incide molto di più del costo del materiale, essendo correlato al numero di unità da impiegare per numero di pazienti.

Basti pensare che in ambito domiciliare vengono trattate ferite per anni perché non riescono a guarire con le medicazioni tradizionali. Per diagnosi errata o scarsa professionalità o conoscenza, il paziente è costretto a convivere con una ferita anche per decenni.

Questo comporta notevoli implicazioni in termini di costi, specialmente quanto risultino necessario frequenti interventi del personale per i cambi della medicazione. ${ }^{2}$

Studi randomizzati e controllati avvalorano questa affermazione confrontando la NTP con altre medicazioni sulle ulcere del piede diabetico, sulle ulcere venose degli arti inferiori, su ulcere da pressione e considerando che mediamente la medicazione della NTP viene cambiata, in ferite non infette, ogni 48-72 ore, al contrario della medicazione standard.

Spesso le medicazioni sono ritenute equivalenti, considerando il prezzo come unica variabile.

Tutto ciò è sbagliato, in quanto ogni medicazione ha una sua proprietà, caratteristica e può determinare benefici sia per l'operatore che per il paziente e quindi una scelta eseguita sul costo unitario, comporta un aumento delle risorse e utilizzo di prodotti aggiuntivi per integrare la performance delle medicazioni a basso costo. ${ }^{2}$

Uno studio del Servizio Sanitario del Canada, ha dimostrato che passare da medicazioni buone a medicazioni avanzate, insieme a una buona pratica, ha ridotto il tempo di guarigione e dei costi complessivi nonostante il costo del materiale fosse aumentato. ${ }^{3}$

Apelqvist et al. ${ }^{4}$ hanno utilizzato le medicazioni della NTP su 162 pazienti, che avevano subito amputazione del piede diabetico, che venivano cambiate dopo 2 giorni, e dopo un confronto con uso di medicazioni standard, hanno rilevato che il tempo medio di guarigione è stato di $56 \mathrm{gg}$ dei pazienti trattati con la NTP rispetto ai $77 \mathrm{gg}$ trattati con altre medicazioni. Oltre a ciò, anche il tasso di guarigione è risultato più alto, il $56 \%$ vs $39 \%$ rispetto a terapia standard (Figura 1).

Smith ha revisionato uno studio sull'efficacia della NTP in confronto agli alginati e idrocolloidi nelle lesioni da pressione. ${ }^{5}$ Egli ha dimostrato una guarigione delle ferite trattate con NTP del $93 \%$ rispetto quelle trattate con idrocolloidi del $63 \%$. Le lesioni trattate con la NTP hanno raggiunto una

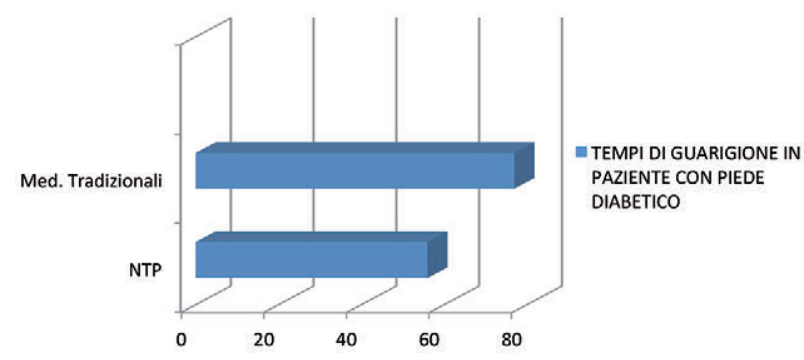

Figura 1. Tempi di guarigione in paziente con piede diabetico. 
guarigione soddisfacente entro 4 settimane $v s$ le cinque e dieci settimane con alginati-idrocolloidi (Figura 2).

Quindi, esaminando i dati, oltre al costo bisognerebbe valutare altri fattori come frequenza dei cambi medicazione, percentuale di guarigione grazie alle nuove tecnologie o nuovi materiali, percentuali di eventuali ricoveri, ecc.

Considerando solo i costi delle medicazioni e del materiale utilizzato nel Wound Care non ha molto successo.

A questo proposito uno studio ${ }^{6}$ è stato eseguito dal Dott. Carnali e la sua equipe presso il centro di Ferite difficili di Fabriano, nel trattare le cisti pilonidali con terapia a pressione negativa.

Per cisti pilonidale o sinus pilonidalis si intende una formazione cistica contenente spesso peli (pili nidus). È conosciuta anche come cisti sacro-coccigea perché situata esclusivamente in questa regione e non va confusa con le patologie fistolose e suppurative a partenza dal canale anale (dalle ghiandole di Hermann e Desfosses).

La procedura che sembra portare a un tasso di guarigione maggiore sembra essere costituito dall'intervento chirurgico, che consiste nell'exeresi chirurgica estesa sino al piano presacrale e comprendente, oltre alla cisti primaria, tutti i tramiti fistolosi resi visibili al chirurgo con opportune metodiche di evidenziazione come l'uso di coloranti vitali (es. blu di metilene) che introdotti a pressione negli orifizi fistolosi nell'immediato preoperatorio rendono identificabile i loro tragitti.

Difficilmente guariscono per prima intenzione (ferita chiusa), nella maggior parte delle volte possiamo assistere a una deiscenza della ferita chirurgica, con tempi molto più lunghi nella sua guarigione o con infezioni della ferita.

Ciò ci fa capire che il paziente dovrà essere medicato giornalmente per la quantità di essudato, la contaminazione che può avvenire con feci, peculiarità del paziente (obesità, ipertricosi, iperidrosi, ecc.) parametri da non sottovalutare nel post chirurgico.

Quindi, l'obiettivo è quello di mantenere la ferita pulita, buon controllo dei fluidi con controllo del cattivo odore.

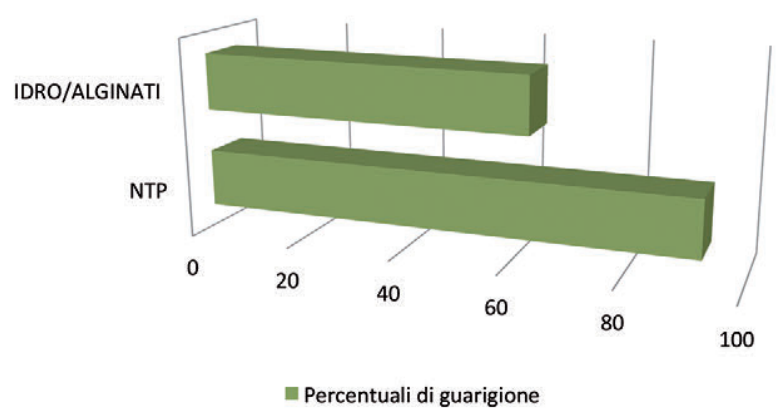

Figura 2. Percentuali di guarigione dei pazienti con lesioni da compressione.
Questo comporta per il paziente numerosi cambi della medicazione che condizionano pesantemente la sua vita e la relazione con altri, nonché ad accedere in ambulatorio giornalmente.

L'U.O. chirurgica di Fabriano ha pensato di utilizzare la NTP nella gestione delle cisti pilonidali nel post-operatorio trattate per deiscenza della ferita o perché operate con tecnica aperta.

Così, hanno potuto valutare: ${ }^{6}$

i) Tempi di guarigione;

ii) Qualità di vita dei pazienti;

iii) Impegno delle risorse umane;

iv) Impatto sociale ed economico.

\section{Tempi di guarigione}

Tutti i pazienti sono guariti entro un tempo di 40-45 giorni.

Come primo approccio hanno utilizzato la NTP, per un maggior controllo degli essudati, mantenimento della pulizia del letto di ferita ed il riempimento della perdita di sostanza.

In un secondo tempo, la completa gestione della ferita con l'impiego di medicazioni avanzate.

\section{Qualità della vita}

Dopo una settimana, il controllo dell'essudato ha permesso ai pazienti il reinserimento nel contesto sociale e lavorativo. Inoltre, il dolore era scomparso.

\section{Analisi delle risorse, sanitarie e non, impiegate nel processo di cura}

L'analisi tiene conto dei costi medi di detersione, trattamento con NTP, trattamento con medicazioni avanzate, trattamento con medicazioni tradizionali, accesso ambulatoriale giornaliero o bisettimanale o trisettimanale, costo orario infermiere, ore lavoro/studio perse, impegno del care-giver.

Da questo è emerso quali siano i costi sanitari ma è stato sottolineato quanto le risorse umane siano di gran lunga più costose nel processo di presa in carico e cura.

Dai calcoli è emerso inoltre come l'accorciamento dei tempi di trattamento con tecnica di NTP e medicazioni avanzate comporti di per sé un risparmio per il Servizio Sanitario Nazionale.

Dalla valutazione eseguita dal 2007-2012 i costi sono risultati inferiori ai $2000 €$, mentre l'approccio tradizionale ha comportato un costo di $3000 €$.

La maggior spesa a cambio, dovuta a materiali più costosi, è più che compensata dal minor numero di accessi ambulatoriali necessari (22 contro 83 medi stimati) e al conseguente minor consumo di tempo medico ed infermieristico.

I vantaggi economici appaiono ancor più significativi 
se sommiamo il costo di guarigione, variabile nel trattamento con NTP e medicazioni avanzate fra $1031 €$ e $1134 €$, e quello della medicazione tradizionale che si mantiene superiore ai $3000 €$.

\section{Impatto sociale ed economico}

Lo studio ha fatto una stima di quelli che possono essere i costi sociali a carico del paziente, intesi come tempo di lavoro perso e costi sostenuti per l'assistenza, senza entrare nel campo dei cosiddetti costi umani, vale a dire tutto quello che riguarda la sofferenza e il disagio patito.

Il risultato emerso è che, con la tecnica NTP + medicazioni avanzate, la guarigione in 45 giorni medi comporti dei costi sociali inferiori a $2000 €$, mentre nel caso di utilizzo di medicazioni tradizionali questi costi superano anche gli $11000 €$.

Sulla stima di questi numeri si può discutere a lungo, ma quello che emerge è che:

i) Questi costi esistono e in qualche modo vanno considerati in quanto, pur non impattando direttamente sul SSN, sicuramente impattano sul PIL del paese.

ii) Il protrarsi del periodo di cura porta a un enorme aumento anche di questi costi (oltre che di quelli a diretto carico del SSN) (Figura 3).

Il più rapido reinserimento nel contesto sociale ed economico espresso in un minor numero di giornate lavorative/studio perse ci permette di affermare che: con il tipo di percorso gestionale descritto è possibile oltre che diminuire il costo per il SSN ridurre in modo significativo il disagio sociale, migliorare la qualità del livello di autonomia e ridurre il ricorso a caregivers diversi (es. genitori, coniuge, ecc.) per la gestione del proprio bisogno sanitario.

Questo significa ridurre i costi economici di contesto che normalmente non sono considerati quali costi, nella mera visione sanitaria del problema.

Un altro studio ${ }^{7}$ fornito dal Dott. Lauletta sui costi beneficio dalla NTP ha dimostrato che: Il costo delle medi-

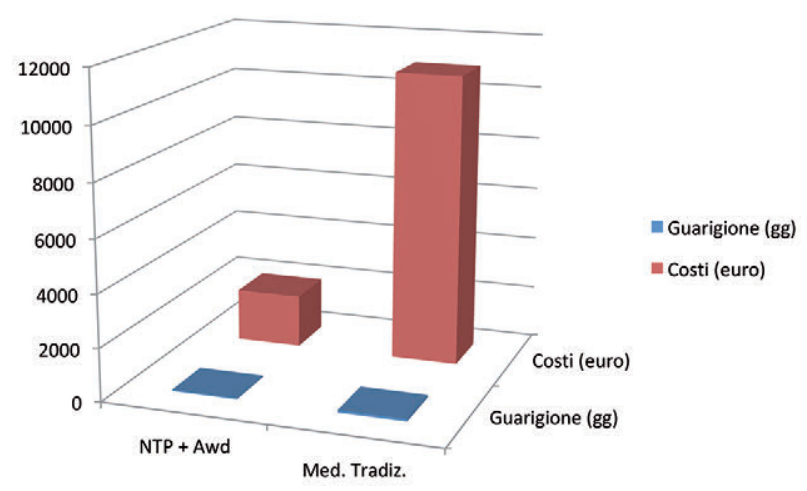

Figura 3. Tempi di guarigione e costi. cazioni avanzate per 4 settimane ha un costo di circa 120 euro mentre il costo della NTP sempre per 4 settimane ammonta a 1200 euro.

Però, lo studio ha dimostrato che il tempo di guarigione con NTP porta a minor accessi e a una ridotta ospedalizzazione per un totale di circa 1697 euro.

Le medicazioni avanzate aumentano il tempo di guarigione, maggiori accessi, e avvolte ospedalizzazioni per un totale di circa 3580 euro.

Quindi, si è calcolato che il costo totale per la gestione di ulcere ha un costo di medicazione pari al $20 \%$, con costi di accessi al 30\%, costi di ospedalizzazione pari al 30\% e da aggiungere costi per eventi avversi che ammontano al $20 \%$ (es. amputazione di gamba).

Quindi, da questi studi emerge che:

i) I pazienti trattati con NTP guariscono prima;

ii) Minor accessi infermieristici;

iii) Minor frequenza di ospedalizzazione;

iv) Minor eventi avversi.

Di fatto, analizzando i dati in base alle evidenze scientifiche ed incrociando i dati dei costi risulta evidente che il rapporto costo/beneficio alla guarigione della lesione è a favore del trattamento con NTP.

La NTP migliora in modo significativo anche la qualità della vita (studio comparativo basato su questionari, condotto in 98 centri, con comparazione dei dati raccolti prima e dopo la NTP). ${ }^{7}$

Questo studio rileva come le lesioni hanno un impatto negativo sulle funzioni psicologiche e sociali del paziente.

Convivere con una ferita influisce negativamente su tutti gli aspetti della vita del paziente.

Una persona che non può lavorare è un soggetto che può avere difficoltà in ambito finanziario e questo provoca rabbia, frustrazione, mancanza di autostima.

Il costo umano delle ferite si misura in dolore, disagio, imbarazzo, ansia, degenze ospedaliere prolungate, morbidità cronica o addirittura morte.

Le cosiddette lesioni difficili vanno affrontate con professionalità e umanità nei confronti del paziente.

Oltre ai costi, non dimentichiamo il Diritto alla Salute, diritto fondamentale di ogni persona.

La convivenza con lesioni di difficile guarigione porta a una serie di problemi psicosociali, che se non adeguatamente affrontati e gestiti, possono indurre una risposta negativa al trattamento ed aggravare le difficoltà di guarigione.

Penso che la maggior parte di queste sofferenze possono essere evitate.

L'impatto che le lesioni determinano sulla qualità di vita del paziente è debilitante.

L'impatto che una lesione comporta sull'individuo colpisce sfera occupazionale, sociale, economica e psicologica.

Molti pazienti possono vivere la cronicità come una 
sfida, cercando di vivere la loro quotidianità, ma altri si ritirano limitando i loro contatti fino a che la situazione migliora.

\section{DISCUSSIONE E CONCLUSIONI}

L'introduzione della terapia a pressione topica negativa ha creato nuove possibilità per la gestione di molte differenti tipologie di lesione.

Quando si prende in considerazione la terapia a pressione topica negativa, la classificazione del tipo di ferite acute e croniche di qualsiasi eziologia è per molti aspetti irrilevante, poiché si richiede piuttosto una valutazione olistica della causa, analizzando nello specifico lo stato della ferita e le sottostanti condizioni mediche, sociali, che potrebbero influenzare il normale decorso di guarigione.

Si potrà così valutare se sia appropriato impiegare questo tipo di intervento.

Oltre ai vantaggi clinici espressi in precedenza, molti studi rilevano anche dei vantaggi economici.

I risultati ottenuti sostengono infatti che l'utilizzo della NTP accelera il processo di guarigione delle ferite e diminuisce le sostituzioni della medicazione, riducendo così $i$ tempi di degenza ospedaliera e risparmiando sui materiali e sul tempo di assistenza infermieristica.

Uno studio in particolare ha evidenziato le potenzialità del presidio in relazione al trattamento di ulcere da piede diabetico, facendo emergere risultati molto positivi come l'accelerazione della guarigione, la riduzione dei tassi di recidive e di amputazione, complicanza che colpisce frequentemente i pazienti con questo tipo di disturbi.

Un trattamento del genere comporta un cambiamento nella vita del paziente per cui un aspetto fondamentale di cui si dovrà occupare l'infermiere esperto sarà sicuramente quello di individuare le esigenze e le preoccupazioni della persona, riconoscere e discutere i possibili ostacoli alla guarigione, fornire assistenza ricorrendo, dove necessario, ad altri specialisti, occupandosi così del paziente nel suo insieme. Alcune ricerche hanno evidenziato che la convivenza con lesioni di difficile guarigione spesso porta a dover affrontare una serie di problemi psicosociali ad esse strettamente correlati, che, se non adeguatamente affrontati e gestiti, possono indurre una risposta negativa al trattamento ed aggravare le difficoltà di guarigione.

Infine valutando un trattamento con NTP therapy homecare è emerso come il paziente possa vivere in modo più positivo la situazione, qualora il nucleo familiare lo consentisse.

\section{BIBLIOGRAFIA}

1. Leininger BE, Rasmussen TE, Smith DL, et al. Experience with VAC and delayed primary closure of contaminated soft tissue injuries in Iraq. J Trauma 2006;61:1207-11.

2. Hurd T, Zuliani N, Posnett J. Evaluation of the impact of restructuring wound management practices in a community care provider in Niagara, Canada. Int Wound J 2008;5:295-303.

3. Armstrong GDG, Lavery LA, Diabetic Foot Study Consortium. Negative Pressure wound therapy after parial diabetic foot amputation: a multicentre, randomized controlled trial. Lancet 2005:366:1704-10.

4. Apelqvist J, Armstrong DG, Lavery LA, Boulton AJ. Resource utilization and economic costs of care based on a randomized trial of vacuum-assisted closure therapy in the treatment of diabetic foot wounds. Am J Surg 2008;62:164-9.

5. International Consensus: Optimising wellbeing in people living with a wound. 2012. Avaliable from: https://www.woundsinternational.com/resources/details/international-consensus -optimising-wellbeing-in-people-living-with-a-wound

6. Carnali M, Ronchi R, Finocchi L, et al. Studio retrospettivo sull'impiego della Terapia a Pressione Negativa (NPWT) nel trattamento delle cisti pilonidali (sinus pilonidalis) operate con tecnica open o complicate da deiscenza del sito chirurgico per sepsi. Acta Vulnol 2016;14:24-39.

7. Lauletta V. Terapia a pressione negativa nella cura delle lesion cutanee croniche: indicazioni della NPWT, costi e benefici deltrattamento a domicilio. Bari: $12^{\circ}$ Congresso Nazionale AGE; 2016. 